\title{
Clinical Features of Disseminated Intravascular Coagulation With Spontaneous Ruptured Primary Renal Angiosarcoma
}

\author{
Seong Hyeon $\mathrm{Yu}^{1}$, Hyo-Jae $\mathrm{Lee}^{2}$, Taek Won Kang ${ }^{1}$ \\ ${ }^{1}$ Department of Urology, Chonnam National University Hospital, \\ Chonnam National University Medical School, Gwangju, Korea \\ ${ }^{2}$ Department of Radiology, Chonnam National University Hospital, \\ Chonnam National University Medical School, Gwangju, Korea
}

\begin{abstract}
Angiosarcoma is a rare malignant tumor accounting for less than $2 \%$ of all soft tissue sarcomas. Primary angiosarcoma of the kidney is an extremely rare but a highly aggressive tumor with poor prognosis. Patients frequently have symptoms of flank pain, hematuria, or abdominal mass. Surgery is the mainstay of treatment followed by radiation with or without chemotherapy. We describe the case of a 61-year-old man with rupture of a primary renal angiosarcoma with clinical features of disseminated intravascular coagulation (DIC). The patient underwent radical nephrectomy, and DIC including uncontrolled thrombocytopenia was resolved after the operation. We suggest that renal angiosarcoma is a cause of extensive retroperitoneal hematomas with clinical features of DIC. (Korean J Urol Oncol 2019;17:190-194)
\end{abstract}

Key Words: Angiosarcoma $\cdot$ Kidney $\cdot$ Thrombocytopenia $\cdot$ Disseminated intravascular coagulation

Angiosarcoma is a rare malignant neoplasm consisting of vascular spaces lined by endothelial cells, and it accounts for $<2 \%$ of all soft tissue sarcomas. ${ }^{1,2}$ Approximately onethird of angiosarcomas arise in the skin, one-third in the soft tissue, and one-third in other anatomical sites such as the liver, breast, and bone. ${ }^{3}$ Angiosarcoma of the kidney is usually found in metastasis from the skin of visceral primary lesions, while primary angiosarcoma arising in the kidney is extremely rare. ${ }^{4}$ Most cases of primary renal angiosarcoma occur in men aged $50-60$ years, but the etiology is

Received October 8, 2019, Revised November 5, 2019,

Accepted November 6, 2019

Corresponding Author: Taek Won Kang

Department of Urology, Chonnam National University Hospital, Chonnam National University Medical School, 42 Jebong-ro, Dong-gu, Gwangju 61469, Korea

E-mail: sydad@hanmail.net

Tel: +82-62-220-6700, Fax: +82-62-227-1643

ORCID: https://orcid.org/0000-0002-7708-819X unknown. ${ }^{1}$ These tumors are highly aggressive with dismal outcomes and are rarely found in case of extensive retroperitoneal hemorrhage as a result of spontaneous rupture. ${ }^{5}$ In this report, we describe the rupture of a primary renal angiosarcoma with clinical features of disseminated intravascular coagulation (DIC) including uncontrolled thrombocytopenia.

\section{CASE REPORT}

A 61-year-old man was transferred to Chonnam National University Hospital due to uncontrolled thrombocytopenia from a local clinic. He worked at a hospital, and there was no prior exposure to environmental carcinogens. He complained of sudden onset of flank pain on his right side, but an abdominal physical examination revealed no rigidity or distension. Furthermore, he had a history of hypertension,

This is an Open Access article distributed under the terms of the Creative Commons Attribution Non-Commercial License (http://creativecommons.org/licenses/by-nc/4.0/) which permits unrestricted non-commercial use, distribution, and reproduction in any medium, provided the original work is properly cited. 2019 (C) Copyright The Korean Urological Oncology Society and The Korean Prostate Society. All Rights Reserved. 
diabetes mellitus, unstable angina pectoris, and dyslipidemia.

At a local clinic, his routine laboratory test results were as follows: white blood cell count, $7.5 \times 10^{3} / \mathrm{mm}^{3}$; hemoglobin, $8.4 \mathrm{~g} / \mathrm{dL}$; platelet count, $69 \times 10^{3} / \mathrm{mm}^{3}$; and creatinine level, $1.3 \mathrm{mg} / \mathrm{dL}$. Multiphase computed tomography (CT) imaging of his abdomen revealed extensive retroperitoneal hemorrhage on his right side, which was thought to be due to a spontaneous rupture of a renal tumor. The huge exophytic mass in the right kidney, measuring $16 \mathrm{~cm}$ in diameter, showed peripheral nodular enhancement (Fig. 1). He underwent an emergency angioembolization at the local clinic. After that, his vital signs were stable; however, thrombocytopenia persisted despite multiple platelet transfusions.

At our hospital, his initial routine laboratory test results were as follows: white blood cell count, $5.1 \times 10^{3} / \mathrm{mm}^{3}$; hemoglobin, $11.0 \mathrm{~g} / \mathrm{dL}$; platelet count, $74 \times 10^{3} / \mathrm{mm}^{3}$; creatinine level, $1.14 \mathrm{mg} / \mathrm{dL}$; prothrombin time, 11.8 seconds; international normalized ratio, 1.02; activated partial thromboplastin time, 32.7 seconds; fibrinogen level, $163.6 \mathrm{mg} / \mathrm{dL}$; fibrinogen degradation products (FDP) level, $125.7 \mu \mathrm{g} / \mathrm{mL}$; and d-dimer level, $35.2 \mathrm{mg} / \mathrm{dL}$. Laboratory test results indicated clinical features of DIC.

Although CT findings of local clinic were already existed, additional magnetic resonance imaging (MRI) was performed to differentiate between benign and malignant tumors including renal hemangioma, renal cell carcinoma, and other hypervascular tumors. MRI revealed a huge hypervascular tumor with an extensive intratumoral hemorrhage. The tumor had high signal intensity on T2-weighted images. Peripheral enhancement was seen in the arterial phase, and centripetal enhancement gradually increased in the delayed phase (Fig. 2). No additional masses were found in the remaining abdomen.

The patient underwent radical right nephrectomy via the transperitoneal approach. A huge mass with extensive hemorrhage was found in the right kidney, which extended up to the perirenal space. The tumor was also observed around the renal hilum. Histologically, complex anastomosing channels with atypical proliferation of endothelial cells were observed. Immunohistochemical stains were positive for
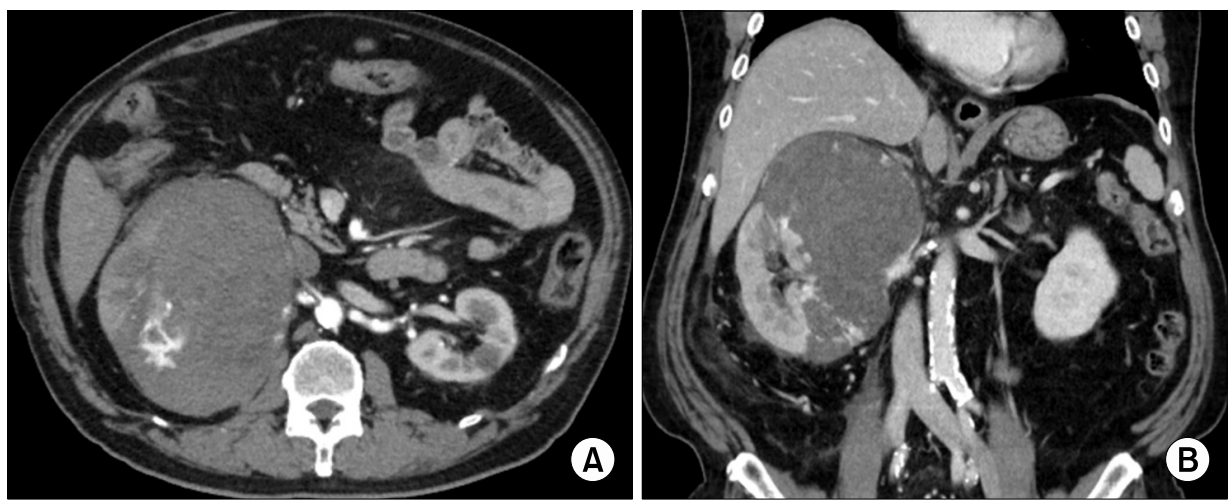

Fig. 1. Computed tomography images revealed an extensive retroperitoneal hemorrhage and huge exophytic mass in the right kidney, which shows peripheral nodular enhancement. (A) Axial image in the arterial phase. (B) Coronal image.
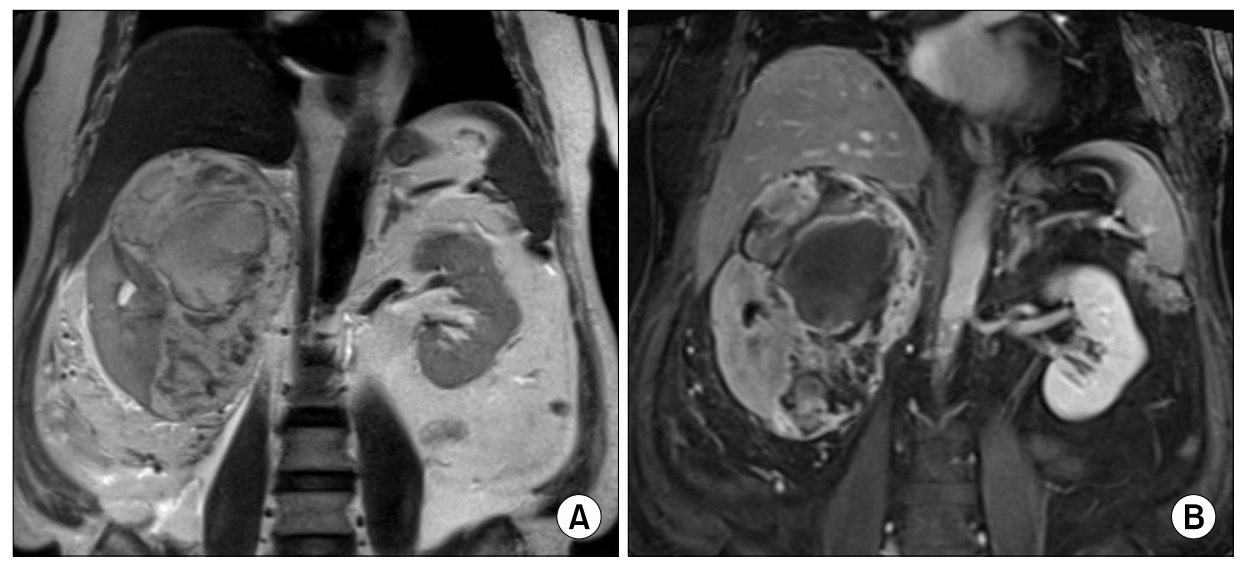

Fig. 2. Magnetic resonance images reveal a huge hypervascular tumor with an extensive intratumoral hemorrhage, which shows strong enhancement on the peripheral area. (A) Coronal T2-weighted image. (B) Contrast-enhanced T1-weighted image. 

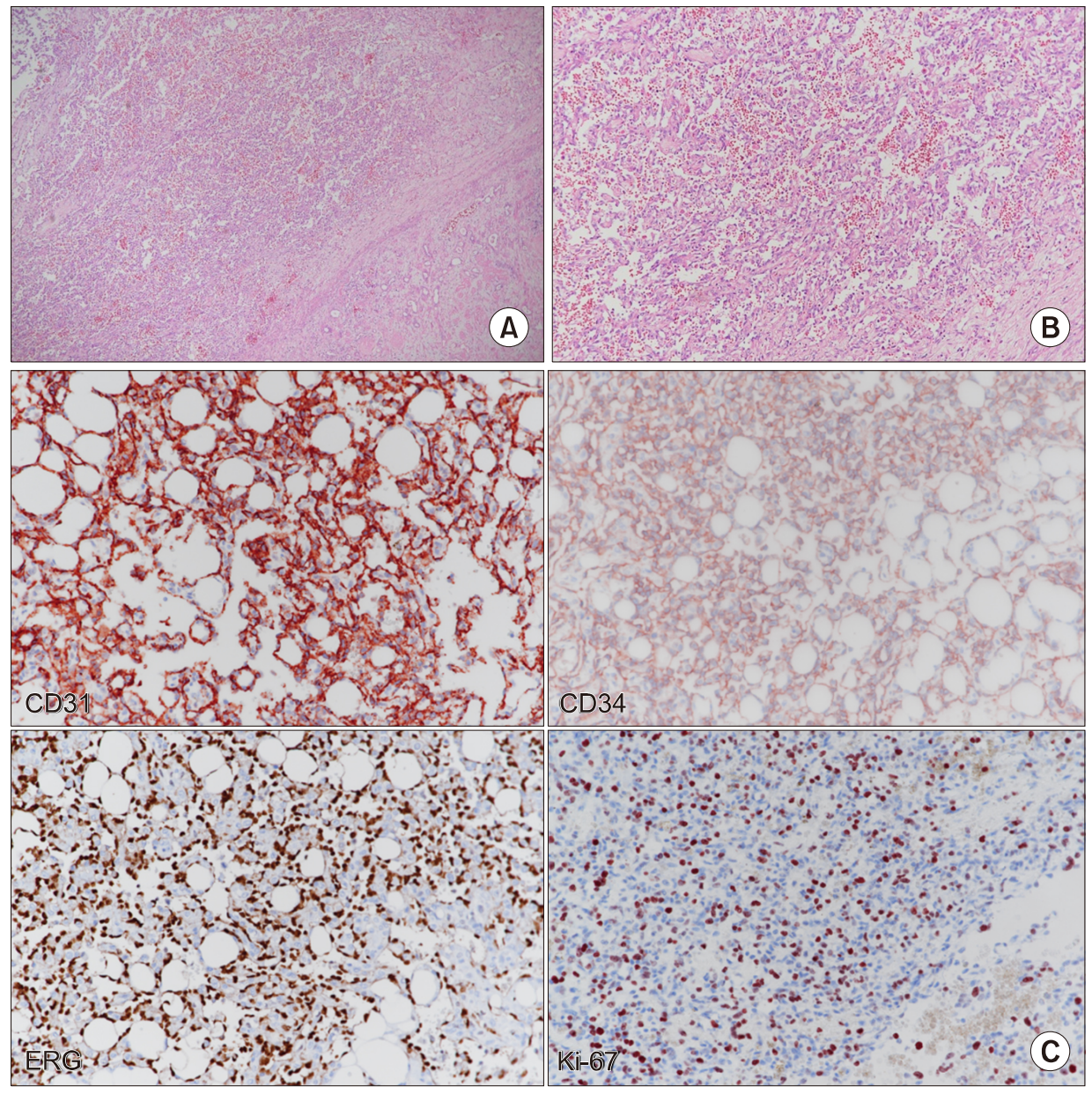

Fig. 3. (A) Histological sections showing the hematoma $(\mathrm{H} \& \mathrm{E}, \times 40)$ and (B) complex anastomosing channels with atypical proliferation of endothelial cells $(\mathrm{H} \& \mathrm{E}, \times 100)$. (C) Positive immunohistochemical staining of the angiosarcoma for CD31, CD34, ERG, and Ki-67.
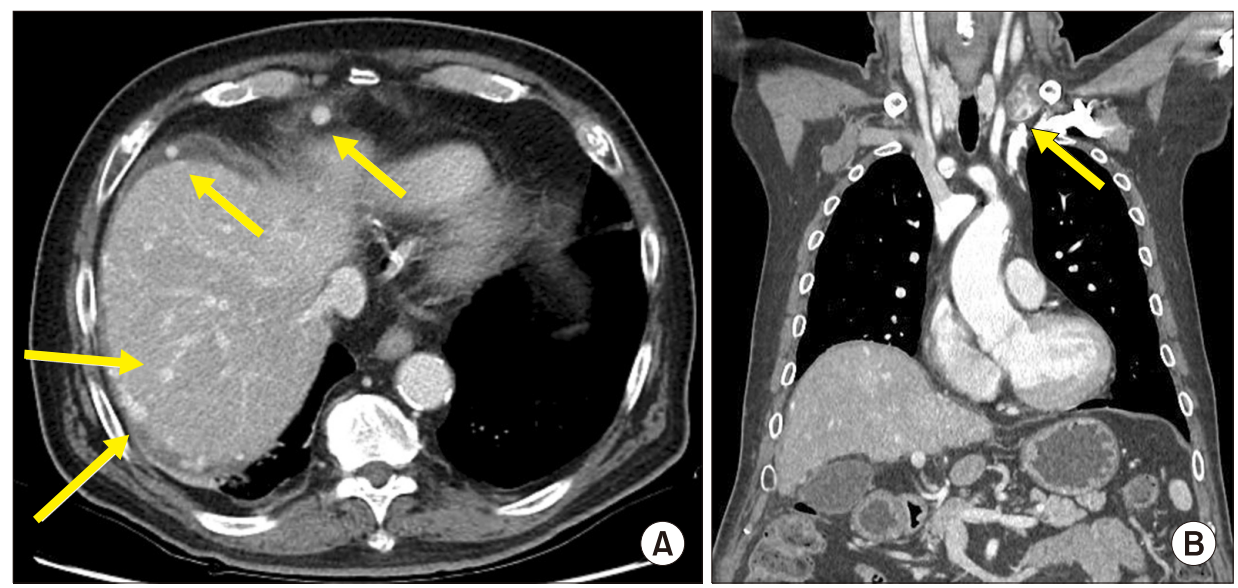

Fig. 4. Computed tomography images. (A) Multiple hypervascular hepatic metastases and peritoneal seeding in perihepatic space (arrows). (B) Left supraclavicular metastatic lymph node (arrow).

ERG, CD31, CD34, and Ki-67 (Fig. 3). A final diagnosis of primary renal angiosarcoma was made based on the histological features.

After surgery, uncontrolled thrombocytopenia were resolved without additional transfusion. DIC profile including fibrinogen, FDP and d-dimer level was also stabilized. The patient underwent adjuvant radiotherapy (OP site, intensity-modulated radiation therapy, 6,300 cGy/30 fractions) and paclitaxel-based chemotherapy (Paclitaxel $100 \mathrm{mg} / \mathrm{m}^{2}$ D1, D8, D15 every 4 weeks) in oncologic department. However, 4 months later, left supraclavicular lymph node, liver, and peritoneal metastases were observed via a CT 
scan (Fig. 4). At 11 months after surgery, the patient is still alive and undergoing palliative treatment.

\section{DISCUSSION}

Primary renal angiosarcoma is an extremely rare malignant neoplasm, and approximately 64 cases have been described to date in the literature. ${ }^{1}$ Although these tumors predominantly occur in men aged 50-60 years, there has been no definitive evidence demonstrating that androgen plays a role. ${ }^{1,2}$ Specific risk factors for primary renal angiosarcoma are not known, while angiosarcomas arising in other sites have been reported to associate with predisposing factors including exposure to arsenic, polyvinyl chloride, thorium dioxide (Thorotrast), radiation, and chronic lymphedema. ${ }^{1,3,6}$ In the present case report, the patient was a 61-year-old man; however, he was not exposed to any of the aforementioned risk factors.

Patients frequently have symptoms, and flank pain is the most common initial presentation. Other clinical symptoms include macrohematuria and/or microhematuria, abdominal mass, weight loss, dizziness, fatigue, and fever. ${ }^{1,3}$ Rarely, if the tumor has a tendency to bleed, patients may present with extensive retroperitoneal hematoma as a result of spontaneous rupture. ${ }^{5}$

Primary renal angiosarcoma has no specific radiologic findings. The tumors are usually large, ranging from 3.7 to $30 \mathrm{~cm}$ in size, and present as a hypodense mass with variable peripheral enhancement on a CT scan. ${ }^{1}$ However, the distinction between malignant lesions of the kidney by only imaging studies before surgery is very challenging. Renal cell carcinoma can also be vascular, and both tumors may appear as large necrotic masses. ${ }^{6}$ In contrast to renal cell carcinomas, most renal angiosarcomas develop into metastatic disease at diagnosis or shortly after. ${ }^{3}$ Our patient had no detectable metastases at diagnosis, which was probably due to the relatively early spontaneous rupture of the tumor.

Immunohistochemically, most angiosarcoma tumor cells stained positively for at least on endothelial cell marker (CD34, CD31, ERG, FLI1, and Factor VIII-related antigen). ${ }^{1,2}$ In contrast, angiosarcoma tumor cells are typically negative for cytokeratin. HHV-8, a marker for Kaposi sarcoma is typically negative. ${ }^{1}$ Immunohistochemical results of our patient were not positive for $\mathrm{HHV}-8$, but positive for
ERG, CD31, and CD34. Therefore, we made a diagnosis of renal angiosarcoma based on immunohistochemical stains.

To date, there have been no specific treatment guidelines for renal angiosarcoma due to the rarity of the tumor. Considering the results of several reports, surgery remains the most effective treatment option. Subsequently, radiotherapy and/or chemotherapy can be administered considering the disease state and clinical condition of patients. ${ }^{1,3}$ However, these therapies have limited success, and local recurrence and distant metastasis commonly occur. In the present case, our patient underwent radical nephrectomy followed by adjuvant radiotherapy and paclitaxel-based chemotherapy. However, multiple liver metastases, left supraclavicular lymph node metastasis, and peritoneal seeding were detected at 5 months after surgery.

The prognosis of renal angiosarcoma is very poor, resulting in high rates of tumor-related death. According to several reports, a large tumor size $(>5 \mathrm{~cm})$ is the most important prognostic factor, and other poor prognostic factors include high tumor grade, head and neck location, margin status, epithelioid histologic component, retroperitoneal location, older patient age, and higher Ki67 values $(\geq 10 \%)$. In the present case, a huge mass that was $16 \mathrm{~cm}$ in diameter in the right kidney was detected by CT scans. Moreover, our patient had other poor prognostic factors including positive margin status, retroperitoneal location, and higher Ki67 values $(30 \%)$.

Angiosarcomas can be associated with consumptive coagulopathy. There have been some reports of angiosarcomas originating in the liver, breast, and mediastinum with clinical features of DIC. ${ }^{7}$ However, to our knowledge, there have been no reports of renal angiosarcoma. Overall, we describe a case of primary renal angiosarcoma with clinical features of DIC. Our patient underwent radical nephrectomy, and DIC including uncontrolled thrombocytopenia was resolved after surgery. This may be the first report of renal angiosarcoma with clinical features of DIC. However, the mechanism remains unclear, and consumptive coagulopathy may be associated with extensive retroperitoneal hemorrhage caused by spontaneous rupture of the tumor. Further studies are needed to better understand the mechanism of association with consumptive coagulopathy and renal angiosarcoma. 
In conclusion, primary renal angiosarcoma is a highly aggressive tumor with poor outcomes and may be a cause of extensive retroperitoneal hematomas. We suggest being mindful of the fact that renal angiosarcomas can be associated with consumptive coagulopathy.

\section{CONFLICT OF INTEREST}

The authors claim no conflicts of interest

\section{REFERENCES}

1. Omiyale AO, Carton J. Clinical and pathologic features of primary angiosarcoma of the kidney. Curr Urol Rep 2018; 19:4.

2. Iacovelli R, Orlando V, Palazzo A, Cortesi E. Clinical and pathological features of primary renal angiosarcoma. Can
Urol Assoc J 2014;8:E223-6.

3. Leggio L, Addolorato G, Abenavoli L, Ferrulli A, D'Angelo C, Mirijello A, et al. Primary renal angiosarcoma: a rare malignancy. A case report and review of the literature. Urol Oncol 2006;24:307-12.

4. Johnson VV, Gaertner EM, Crothers BA. Fine-needle aspiration of renal angiosarcoma. Arch Pathol Lab Med 2002; 126:478-80

5. Aksoy Y, Gürsan N, Ozbey I, Biçgi O, Keleş M. Spontaneous rupture of a renal angiosarcoma. Urol Int 2002;68:60-2.

6. Brown JG, Folpe AL, Rao P, Lazar AJ, Paner GP, Gupta $\mathrm{R}$, et al. Primary vascular tumors and tumor-like lesions of the kidney: a clinicopathologic analysis of 25 cases. Am J Surg Pathol 2010;34:942-9.

7. Farid M, Ahn L, Brohl A, Cioffi A, Maki RG. Consumptive coagulopathy in angiosarcoma: a recurrent phenomenon? Sarcoma 2014;2014:617102. 